\title{
Primary gastric melanoma with pancreatic metastasis: a case report
}

\author{
Zhihuan Xiao", Gang Ma\#, Guichen Li, Anjiang Gou, Yuanhong Xu, Shaowei Song, Kejian Guo, Zhe Liu \\ Department of Pancreatic-Biliary Surgery, First Hospital of China Medical University, Shenyang, China \\ \#These authors contributed equally to this work. \\ Correspondence to: Dr. Zhe Liu. 155 North Nanjing St, Shenyang 110001, China. Email: liuzhe4321@126.com.
}

\begin{abstract}
We report an extremely rare case of primary gastric melanoma with pancreatic metastasis. As far as we know, the concept of primary gastrointestinal melanoma is currently controversial, because there are very few reports of primary gastrointestinal melanoma. At the same time, isolated pancreatic metastases are also very rare. The patient was admitted to the hospital with a main complaint of decreased appetite, and then underwent gastroscopy and found a mass in the stomach. The mass was initially diagnosed as poorly differentiated adenocarcinoma following a gastroscopic biopsy. The patient underwent radical total gastrectomy, pancreatic body and tail resection, splenectomy, and Roux-en-Y esophagojejunostomy. After further immunohistochemical examination of the surgically removed tissue, malignant melanoma was diagnosed. The tumor cells were arranged in sheets or nests with infiltrating growth, the cell sizes were inconsistent, nucleoli were obvious, and melanin particles were seen in the cytoplasm of some cells. The tumor cells were positive for MITF and S-100. Detailed systemic and imaging examinations did not find any other primary lesions. The patient denied any melanoma and skin lesion history. We believe this is a manifestation of primary gastric melanoma. We report this rare case of gastric melanoma with the aim of increasing clinicians' awareness of non-cutaneous melanoma and its treatment methods.
\end{abstract}

Keywords: Melanoma; primary gastric melanoma; pancreatic metastasis; case report

Submitted Mar 29, 2021. Accepted for publication May 19, 2021.

doi: $10.21037 /$ apm-21-1170

View this article at: http://dx.doi.org/10.21037/apm-21-1170

\section{Introduction}

Melanoma is a rare malignant tumor that originates from melanocytes. Melanocytes are mostly distributed in the skin, uvea, meninges, and mucous membranes, and the incidence of melanoma in these areas is $91.2 \%$, $5.2 \%, 1.3 \%$, and $2.2 \%$, respectively (1). Gastrointestinal melanoma is very rare, and the main clinical manifestations are weight loss, anemia, loss of appetite, and other nonspecific symptoms that pose a great challenge to early diagnosis. At present, it is believed that most melanomas that appear in the gastrointestinal tract are metastatic, although literature reports on gastrointestinal melanoma are gradually increasing. According to a previous study, 4\% of skin melanoma patients had gastrointestinal metastasis before death, and up to $60 \%$ at autopsy (2). Therefore, a thorough physical examination, imaging examination, and laboratory tests are essential to confirm the diagnosis.
Since gastrointestinal melanoma cannot be distinguished from metastatic melanomas that regress on their own, the concept of primary gastrointestinal melanoma is still controversial. However, there is evidence that there are melanocytes in the anal canal and esophagus $(3,4)$.

We report on a man who had multiple ulcerative lesions in the fundus and body of the stomach with lumps in the body and tail of the pancreas. His main symptoms were decreased appetite and rapid weight loss. Detailed pathological biopsy and immunohistochemistry confirmed that the lesion was melanoma. After thorough systemic and imaging examinations, we found no other primary lesions except for the metastases in the pancreas. We believe that this is a rare manifestation of primary gastric melanoma.

We present the following article in accordance with the CARE reporting checklist (available at http://dx.doi. org/10.21037/apm-21-1170). 

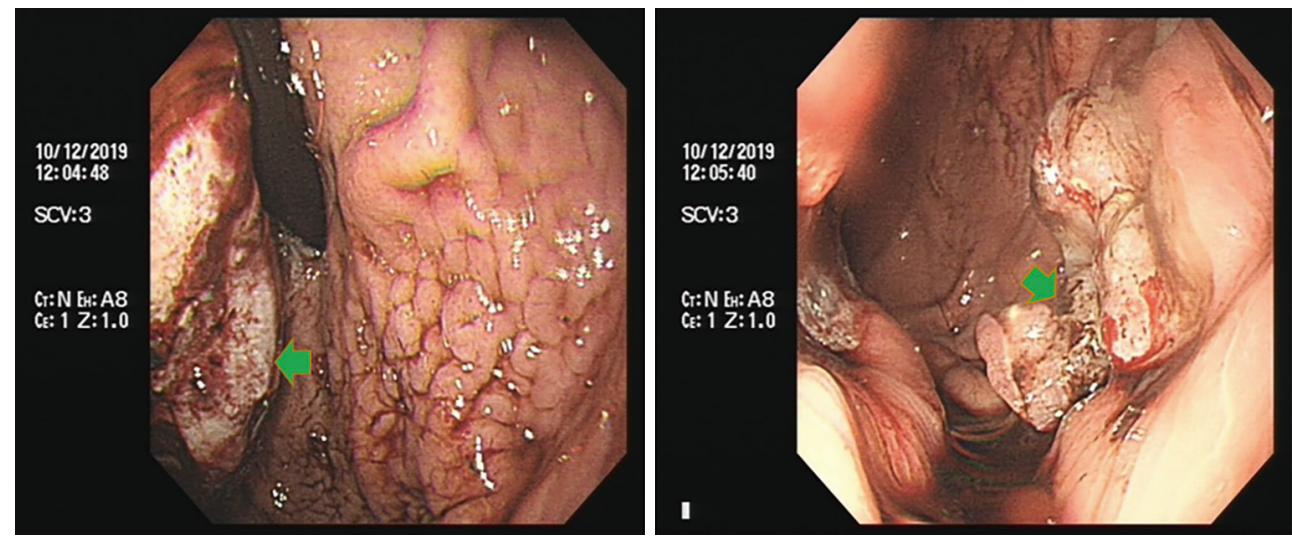

Figure 1 Irregular ulcerative lesions on the fundus and body of the stomach (green arrow).
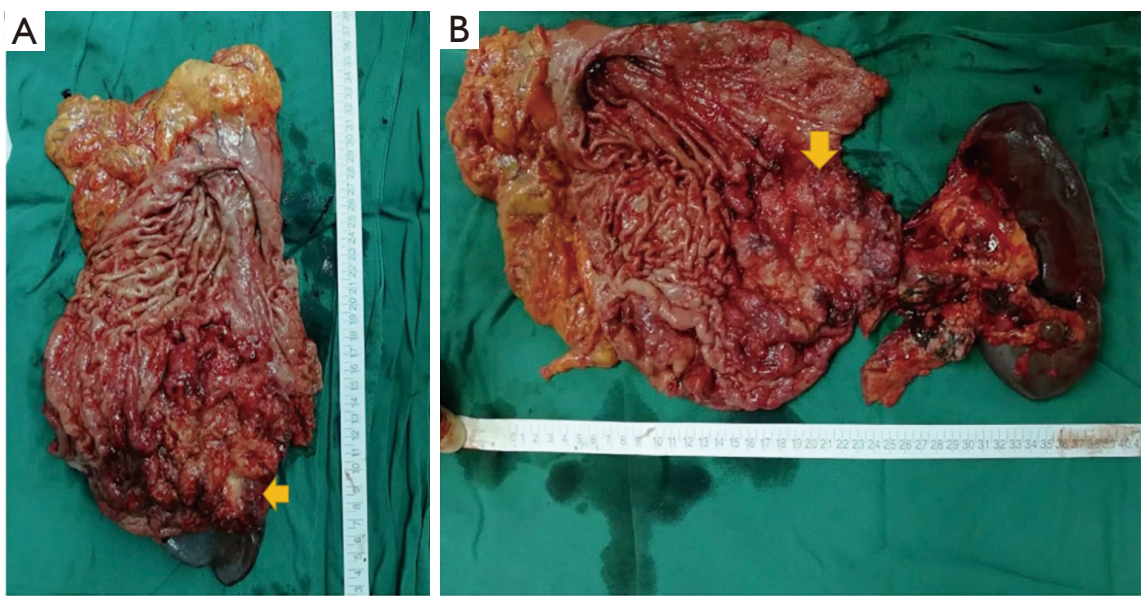

Figure 2 Macroscopic image of tumor cut surface of resected tumor specimen. (A) Ulcers and lumps in the stomach (yellow arrow). (B) The complete specimen removed by surgery. Stomach tumor invades the pancreatic parenchyma (yellow arrow).

\section{Case presentation}

A 65 -year-old man came to our hospital with a 20-day history of decreased appetite, accompanied by flatulence, nausea, and rapid weight loss. The patient had no history of surgery, diabetes, hypertension, coronary atherosclerotic heart disease, hepatitis, tuberculosis, or other medical history. He denied a history of drinking, melanoma, or a family history of cancer. A gastroscopy showed multiple irregular ulcerative lesions in the gastric fundus and body, the largest one being $3.0 \mathrm{~cm} \times 2.5 \mathrm{~cm}$ (Figure 1), and the pathology showed poorly differentiated adenocarcinoma of the fundus of the stomach. On admission, the tumor indicators, including CA19-9 and CEA, were normal. An enhanced CT showed that the gastric fundus was thickened with uneven enhancement, and multiple enlarged lymph nodes were seen around the pancreatic body, the largest of which was $2.1 \mathrm{~cm} \times 2.8 \mathrm{~cm}$. There was a mass shadow on the tail of the pancreas measuring $3.1 \mathrm{~cm} \times 3.9 \mathrm{~cm}$. Its internal density was uneven and liquefied necrosis was observed. According to the patient's clinical manifestations, imaging findings, and gastroscopic pathological results, a preliminary diagnosis of poorly differentiated adenocarcinoma of the stomach with pancreatic metastasis was made. The patient underwent surgical treatment that included radical total gastrectomy, pancreatic body and tail resection, splenectomy, and Roux-en-Y esophagojejunostomy. In Figure 2, the surgically resected tissue can be seen as a $4.0 \mathrm{~cm}$-diameter infiltrating tumor in the fundus of the stomach, and the tumor is seen invading the pancreatic parenchyma. Figure 3 
shows a $3.0 \mathrm{~cm}$ mass on the tail of the pancreas, and the cut surface shows a typical black and white pigmentation. Immunohistochemistry showed that all resection margins

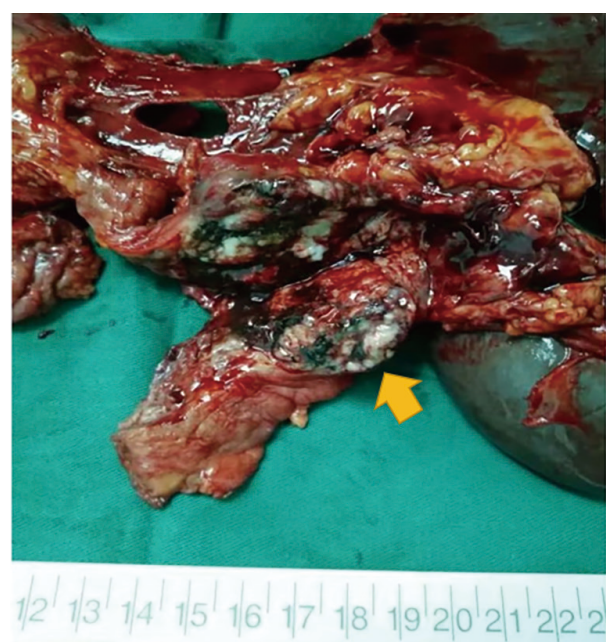

Figure 3 A pancreatic mass with black and white pigmentation on the cut surface (yellow arrow). were negative, and there was no lymph node metastasis. In Figure 4, HE staining shows that the cells are of different sizes, and large nucleoli and melanin particles can be seen in the cells. The immunohistochemistry of the resected tissue was positive for MITF and S-100. Tumor KI67 expression was $70 \%$, confirming the diagnosis of malignant melanoma (Figure 5). Postoperatively, the patient was generally in a stable condition. He was given general supportive treatment because he declined to receive conventional postoperative radiotherapy and chemotherapy. The patient was discharged 16 days later and died 3 months after discharge.

All procedures performed in studies involving human participants were in accordance with the ethical standards of the institutional and/or national research committee(s) and with the Helsinki Declaration (as revised in 2013). Written informed consent was obtained from the patient.

\section{Discussion}

Primary gastrointestinal melanoma is very rare in clinical practice, and part of the reason can be explained by the
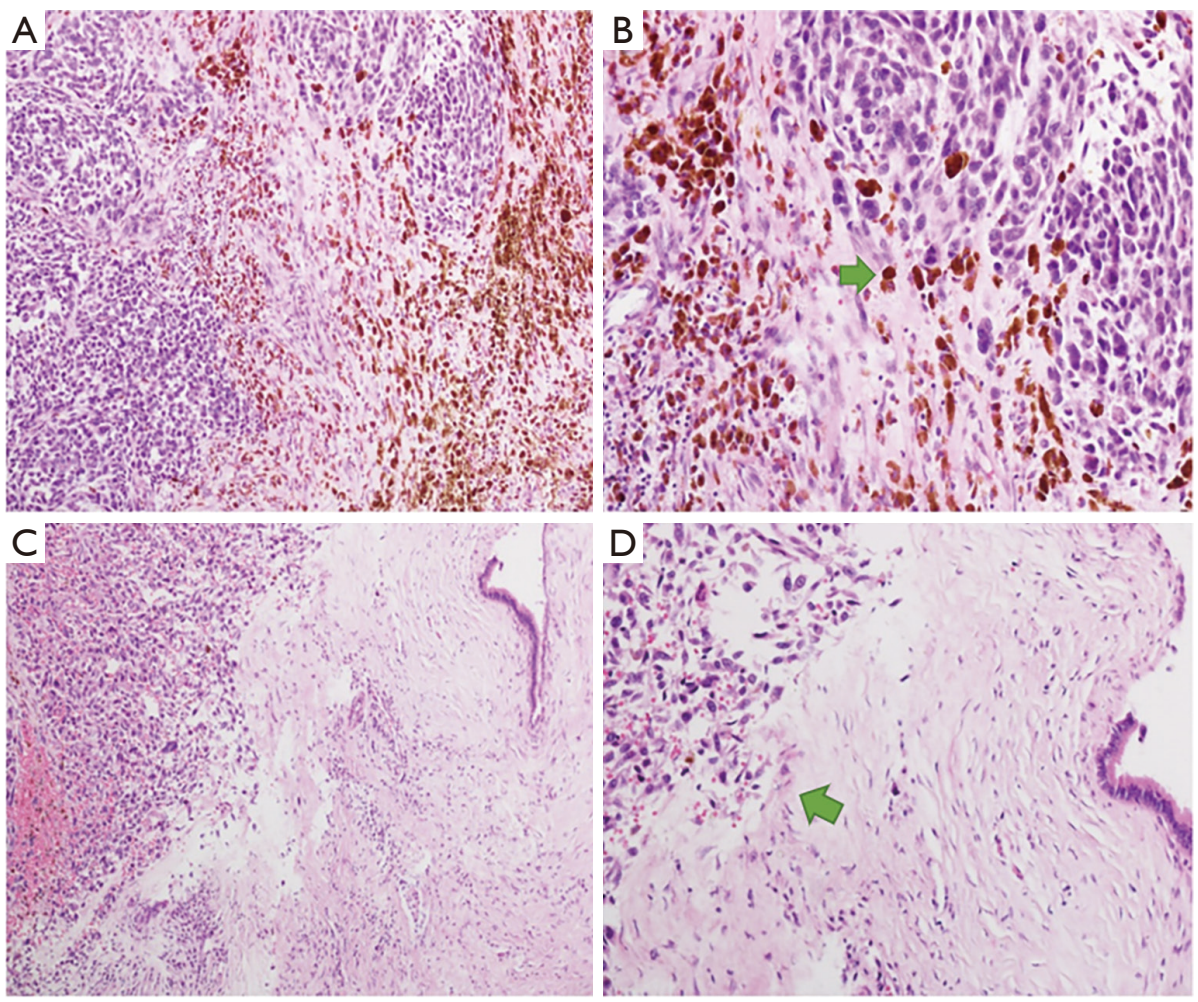

Figure $4 \mathrm{HE}$ staining: Invasive growth of cancer cells, cell sizes are different, nucleoli are obvious, and melanin particles can be seen in some cells (green arrow). (A) $\times 100$ (B) $\times 200$. Tumor cells invade the pancreas, compressing pancreatic ducts $($ green arrow). (C) $\times 100,(D) \times 200$. 

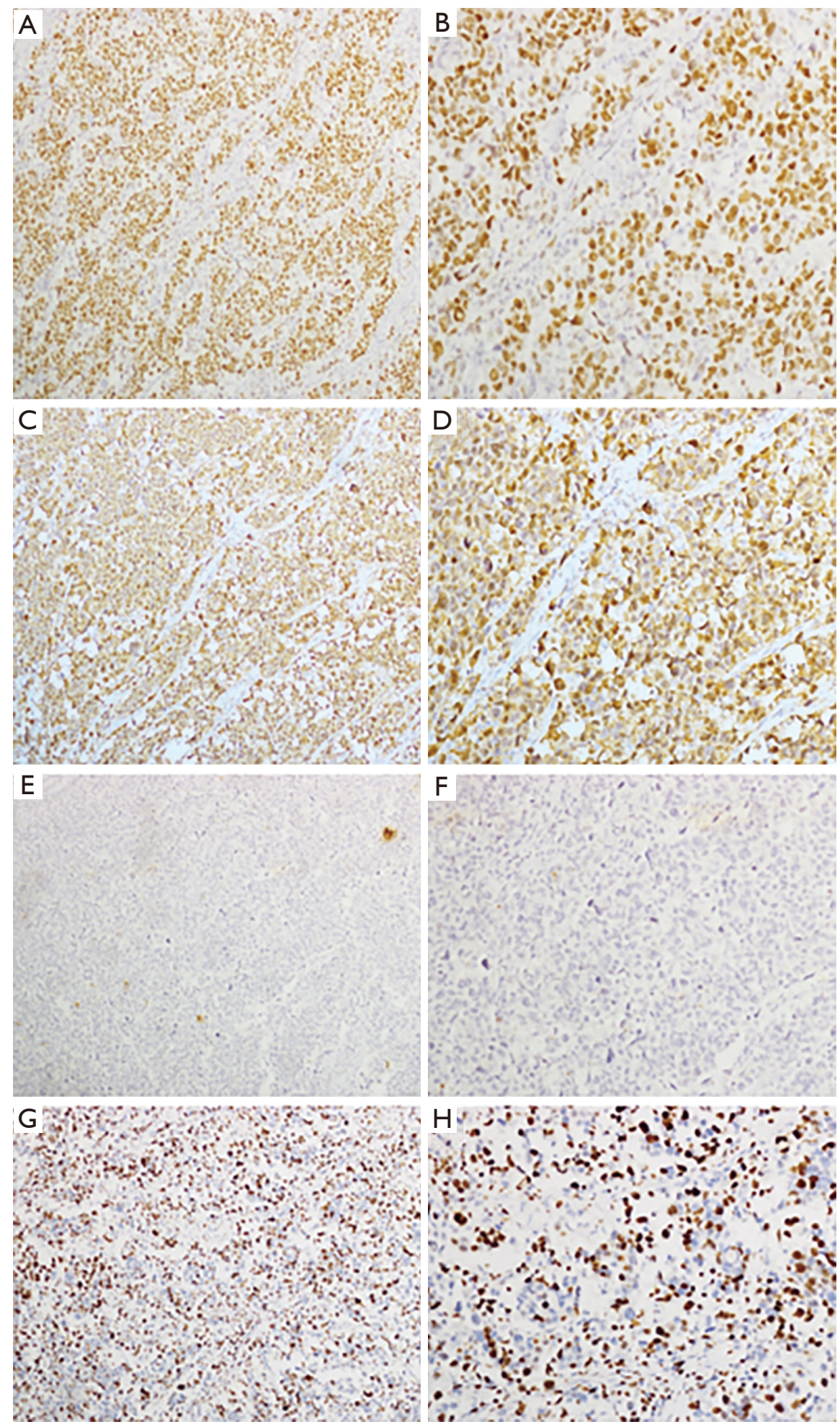

Figure 5 Histopathological appearance of resected tumor. Positive immunohistochemical (IHC) staining of MITF protein (A,B). Positive immunohistochemical (IHC) staining of S-100 protein Positive immunohistochemical (IHC) staining of S-100 protein (C,D). Negative immunohistochemical (IHC) staining of $\mathrm{CK}$ protein (E,F). Immunohistochemical (IHC) staining of KI67 expression is about $70 \%$ (G,H). $(\mathrm{A}, \mathrm{C}, \mathrm{E}, \mathrm{G}) \times 100 ;(\mathrm{B}, \mathrm{D}, \mathrm{F}, \mathrm{H}) \times 200$. 
lack of ultraviolet radiation and the lack of melanocytes in the digestive tract. As far as we know, the vast majority of gastrointestinal melanomas are metastases from skinderived lesions. However, we have found 12 cases of primary gastrointestinal melanoma in the literature (5-16). The pathogenesis and progression mechanism of primary gastrointestinal melanoma is still unclear, but two hypotheses have been proposed. First, the neural crest derivatives obtain the ability to differentiate into melanocytes and undergo malignant transformation, leading to melanoma $(17,18)$. Second, the melanocytes from known sites, such as the esophagus, migrate ectopically to the gastrointestinal tract and undergo malignant transformation.

Diagnosis of gastrointestinal melanoma should also exclude skin melanoma metastasis where the primary lesion has resolved spontaneously. First, a complete patient history must be collected, such as whether skin lesions have been removed or resolved spontaneously, and secondly, extensive dermatological and imaging examinations are also necessary. The typical manifestations of primary gastric melanoma include non-specific symptoms such as anemia and rapid weight loss, so it is difficult to make a clear diagnosis before surgery. A PET-CT scan can clearly show the location of the lesion, the degree of metastasis, and the involvement, which is of great value for confirming the diagnosis (6).

Endoscopic observation of pigmented ulcer lesions often prompts the diagnosis of melanoma. Tissue immunohistochemical staining with HMB-45 and S-100 can confirm the presence of malignant melanocytes and is often used as the gold standard for diagnosis (4). Therefore, gastrointestinal endoscopy is the recommended method for confirming an early diagnosis of melanoma.

Mucosal melanoma is more aggressive and has a worse prognosis than cutaneous melanoma because there is a large supply of lymph and blood vessels in the gastrointestinal mucosa, and the inherently aggressive behavior of melanoma means that it will spread faster (19). Early detection and early surgical intervention are imperative for long-term treatment of mucosal melanoma (9), although the overall prognosis is still not optimistic.

In conclusion, gastric primary malignant melanoma remains a disease that has not been fully studied. Because of its initial non-specific symptoms, it is difficult to make an early diagnosis. Except for surgery, there is also a lack of effective treatment methods for non-cutaneous melanoma. Therefore, there is an urgent need to continue the search for other effective auxiliary or non-surgical treatments for non-cutaneous melanoma when the patient is not suitable for surgery.

\section{Acknowledgments}

Funding: This study was supported by grants from the Liaoning Provincial Department of Education Science Research Project (L2014299) and the National Natural Science Foundation of China (81572360).

\section{Footnote}

Reporting Checklist: The authors have completed the CARE reporting checklist. Available at http://dx.doi.org/10.21037/ apm-21-1170

Conflicts of Interest: All authors have completed the ICMJE uniform disclosure form (available at http://dx.doi. org/10.21037/apm-21-1170). All authors report funding from Liaoning Provincial Department of Education Science Research Project (No. L2014299) and National Natural Science Foundation of China (No. 81572360). The authors have no other conflicts of interest to declare.

Ethical Statement: The authors are accountable for all aspects of the work in ensuring that questions related to the accuracy or integrity of any part of the work are appropriately investigated and resolved. All procedures performed in studies involving human participants were in accordance with the ethical standards of the institutional and/or national research committee(s) and with the Helsinki Declaration (as revised in 2013). Written informed consent was obtained from the patient.

Open Access Statement: This is an Open Access article distributed in accordance with the Creative Commons Attribution-NonCommercial-NoDerivs 4.0 International License (CC BY-NC-ND 4.0), which permits the noncommercial replication and distribution of the article with the strict proviso that no changes or edits are made and the original work is properly cited (including links to both the formal publication through the relevant DOI and the license). See: https://creativecommons.org/licenses/by-nc-nd/4.0/.

\section{References}

1. Chang AE, Karnell LH, Menck HR. The National Cancer Data Base report on cutaneous and noncutaneous 
melanoma: a summary of 84,836 cases from the past decade. The American College of Surgeons Commission on Cancer and the American Cancer Society. Cancer 1998;83:1664-78.

2. Patel JK, Didolkar MS, Pickren JW, et al. Metastatic pattern of malignant melanoma. A study of 216 autopsy cases. Am J Surg 1978;135:807-10.

3. De La Pava S, Nigogosyan G, Pickren JW, et al. Melanosis of the esophagus. Cancer 1963;16:48-50.

4. Clemmensen OJ, Fenger C. Melanocytes in the anal canal epithelium. Histopathology 1991;18:237-41.

5. Grilliot MA, Goldblum JR, Liu X. Signet-ring cell melanoma of the gastroesophageal junction: a case report and literature review. Arch Pathol Lab Med 2012;136:324-8.

6. Augustyn A, de Leon ED, Yopp AC. Primary gastric melanoma: case report of a rare malignancy. Rare Tumors 2015;7:5683.

7. Khaliq A, Siddappa PK, Thandassery RB, et al. Melanoma of stomach. J Gastrointest Cancer 2012;43:630-3.

8. Lagoudianakis EE, Genetzakis M, Tsekouras DK, et al. Primary gastric melanoma: a case report. World J Gastroenterol 2006;12:4425-7.

9. Alazmi WM, Nehme OS, Regalado JJ, et al. Primary gastric melanoma presenting as a nonhealing ulcer. Gastrointest Endosc 2003;57:431-3.

10. Kim NR, Lee WK, Chung DH. Primary gastric melanoma with rhabdoid features: a case report. Korean J Pathol 2013;47:606-9.

Cite this article as: Xiao Z, Ma G, Li G, Gou A, Xu Y, Song S, Guo K, Liu Z. Primary gastric melanoma with pancreatic metastasis: a case report. Ann Palliat Med 2021;10(5):5902-5907. doi: 10.21037/apm-21-1170
11. Ravi A. Primary gastric melanoma: a rare cause of upper gastrointestinal bleeding. Gastroenterol Hepatol (N Y) 2008;4:795-7.

12. Castro C, Khan Y, Awasum M, et al. Case report: primary gastric melanoma in a patient with dermatomyositis. Am J Med Sci 2008;336:282-4.

13. Jelincic Z, Jakic-Razumovic J, Petrovic I, et al. Primary malignant melanoma of the stomach. Tumori 2005;91:201-3.

14. Goral V, Ucmak F, Yildirim S, et al. Malignant melanoma of the stomach presenting in a woman: a case report. J Med Case Rep 2011;5:94.

15. Ishizaki M, Aibara Y, Furuya K. Primary malignant melanoma of the esophagogastric junction: Report of a case. Int J Surg Case Rep 2013;4:700-3.

16. Yamamura K, Kondo K, Moritani S. Primary malignant melanoma of the stomach: report of a case. Surg Today 2012;42:195-9.

17. Krausz MM, Ariel I, Behar AJ. Primary malignant melanoma of the small intestine and the APUD cell concept. J Surg Oncol 1978;10:283-8.

18. Elsayed AM, Albahra M, Nzeako UC, et al. Malignant melanomas in the small intestine: a study of 103 patients. Am J Gastroenterol 1996;91:1001-6.

19. Sachs DL, Lowe L, Chang AE, et al. Do primary small intestinal melanomas exist? Report of a case. J Am Acad Dermatol 1999;41:1042-4.

(English Language Editor: D. Fitzgerald) 